\title{
Posterior suture annuloplasty for functional tricuspid regurgitation
}

\author{
Vakhtang Tchantchaleishvili ${ }^{1}$, Taufiek K. Rajab ${ }^{2}$, Lawrence H. Cohn ${ }^{2}$ \\ ${ }^{1}$ University of Rochester Medical Center, Rochester, New York, USA; ${ }^{2}$ Brigham and Women's Hospital, Harvard Medical School, Boston, \\ Massachusetts, USA \\ Correspondence to: Vakhtang Tchantchaleishvili, MD. University of Rochester Medical Center, 601 Elmwood Ave., Box Cardiac Surgery, Rochester, \\ New York 14608, USA. Email: tchantchaleishvili@gmail.com.
}

\begin{abstract}
Functional tricuspid regurgitation (TR) is primarily caused by enlargement of the tricuspid annulus due to right ventricular dilation, frequently secondary to left sided valvular disease. Early techniques for the treatment of functional TR were introduced by Jerome Kay in 1965 and Norberto DeVega in 1972. Modified suture annuloplasty is a modification of DeVega's semicircular purse string technique, however, it is based on Kay's principle of obliteration of the posterior segment of the annulus only. While ring annuloplasty is the procedure of choice for severe functional TR, posterior suture annuloplasty is a technically simpler option for patients with moderate functional TR.
\end{abstract}

Keywords: Tricuspid valve repair; posterior leaflet plication; bicuspidization; functional tricuspid regurgitation

Submitted Sep 21, 2015. Accepted for publication Jan 31, 2017.

doi: 10.21037/acs.2017.05.04

View this article at: http://dx.doi.org/10.21037/acs.2017.05.04

\section{Introduction}

Functional tricuspid regurgitation (TR) is primarily caused by enlargement of the tricuspid annulus due to right ventricular dilation (1). Frequently this is secondary to left sided valvular disease. Early techniques for the treatment of functional TR were introduced by Jerome Kay in 1965 and Norberto DeVega in 1972. The Kay technique involved obliteration of the posterior tricuspid leaflet by placement of several sutures across the posterior segment of the tricuspid valve annulus (2) (Figure 1). In contrast, the DeVega technique involved annular plication by placement of two semicircular purse string sutures around the tricuspid valve annulus (3) (Figure 2). While both techniques were effective, they were superseded by ring annuloplasty after introduction of the remodeling annuloplasty concept by Alain Carpentier $(4,5)$.

Ring annuloplasty is thought to offer the best longterm outcomes for severe functional TR (6). However, this procedure leads to some prolongation of the operation and cardiopulmonary bypass time. Therefore intervening on moderate functional TR in the context of another cardiac procedure may become a decision making dilemma. For such cases, a modified suture annuloplasty technique was developed at Brigham and Women's Hospital (7). This suture annuloplasty is a modification of DeVega's semicircular purse string technique. However, it is based on Kay's principle of obliteration of the posterior segment of the annulus only.

\section{Operative technique}

The degree and mechanism of TR has to be carefully evaluated with echocardiogram preoperatively as intraoperative TEE in a sedated patient does not accurately demonstrate its true severity. Exposure is typically achieved by median sternotomy, unless dictated otherwise by the primary procedure. Inspection of the heart often shows evidence of TR such as right atrial and ventricular dilation. Our preferred approach is the classic horizontal atriotomy. The right atrium and the tricuspid valvular apparatus are carefully inspected. If a transseptal mitral valve intervention is planned, it is performed at this stage, followed by closure of the interatrial septum.

Then, the severity of tricuspid annular dilatation is assessed. The tricuspid valve leaflets are examined for any evidence of pathology. If the annulus and leaflets are favorable for a repair, a pledget-supported mattress suture 

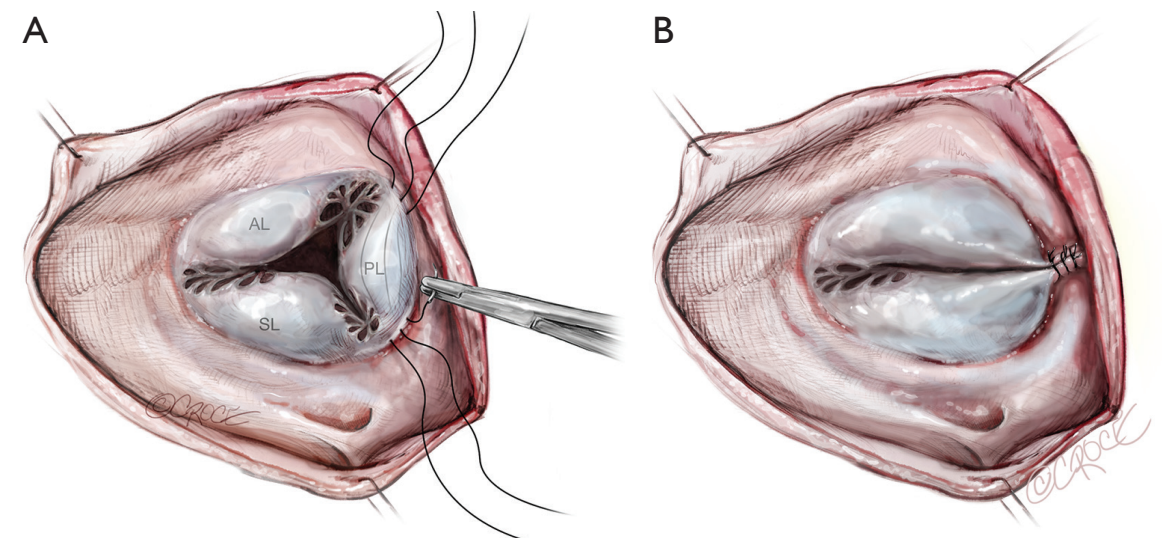

Figure 1 Kay's technique involves an annulorrhaphy of the posterior segment. The resulting perpendicular forces on the tricuspid annulus in Kay's repair obliterate the posterior tricuspid leaflet. AL, anterior leaflet; PL, posterior leaflet; SL, septal leaflet.
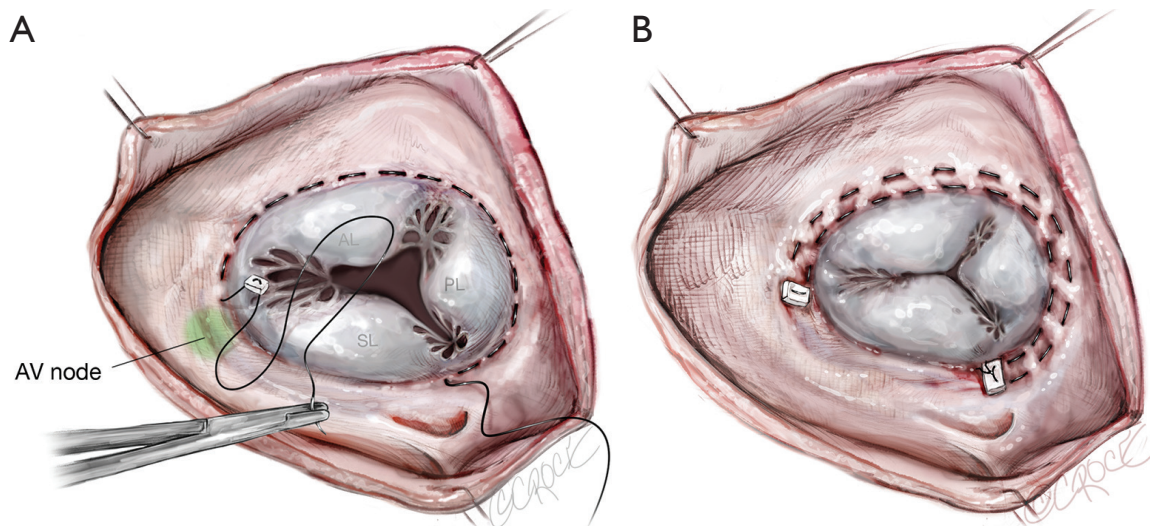

Figure 2 DeVega's technique involves plication of the tricuspid annulus with two semicircular purse string sutures that start in the area of the atrioventricular node and the bundle of his. The resulting centripetal forces in DeVega's repair act on the anterior and posterior segments of the tricuspid annulus to narrow the valve orifice. AL, anterior leaflet; PL, posterior leaflet; SL, septal leaflet.

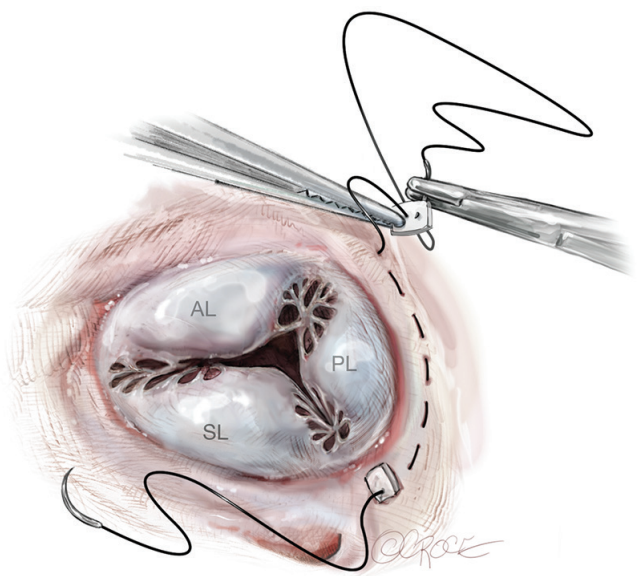

Figure 3 The first arm of the support suture is placed along the posterior annulus. AL, anterior leaflet; PL, posterior leaflet; SL, septal leaflet. of 2-0 Ethibond (Ethicon, Inc., Somerville, NJ, USA) on a large $\mathrm{MH}$ needle is placed from the anteroposterior commissure to the posteroseptal commissure along the posterior annulus (Figure 3). The depth of each bite is 1-2 mm and travel is about $5 \mathrm{~mm}$. The same is repeated for the second arm of the pledgeted suture and, once completed, the second pledget is placed at the posteroseptal commissure (Figure 4). The suture is tied down over the second pledget with an obturator such as a $29-\mathrm{mm}$ valve sizer or the barrel of a $20-\mathrm{mL}$ syringe in the valve annulus that controls the degree of narrowing. This results in a double continuous purse string suture that straddles the posterior segment of the tricuspid annulus (Figure 5).

Finally, the competency of the repaired valve is tested with a saline injection test. 


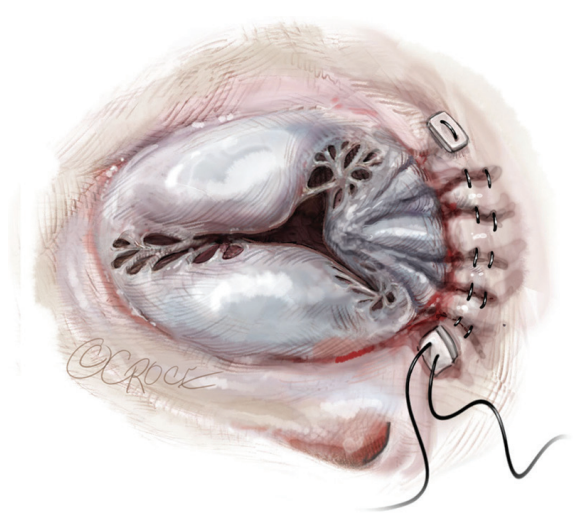

Figure 4 The second arm of the support suture completes the pledgeted mattress.

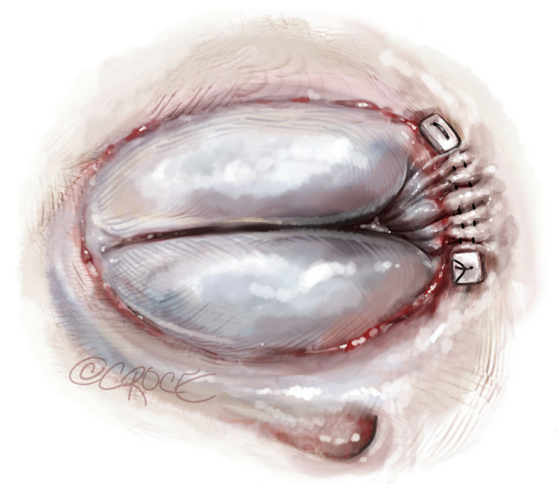

Figure 5 The suture is tied down over an obturator inside the annulus.

The right atrium and the repaired valve are inspected once again. The properly performed annuloplasty gives the repaired valve a bicuspid appearance. The right atrium is then closed and cardiopulmonary bypass is weaned in a standard fashion.

\section{Clinical results}

The largest study of the outcomes after functional TR repair included 2,277 patients, who were followed for 5 years after the index procedure involving functional TR repair concomitant with intervention on either or both leftsided valves. This showed that rigid prosthetic rings had the least increase of moderately severe or severe TR $(12 \%$ at 5 years) followed by flexible prosthetic rings (16\% at 5 years), Kay technique (19\% at 5 years), and DeVega annuloplasty (24\% at 5 years). In general, isolated procedures at the annular level had similar patterns of postoperative TR, including late TR. Overall moderately severe or severe TR was at $17 \%$ by 5 years (8). Analysis of 237 patients at Brigham and Women's Hospital who underwent functional TR repair concomitant with intervention on the left heart demonstrated that posterior suture bicuspidization and ring annuloplasty were equally durable at reducing TR. At 3 years post-operatively, 69\% of patients with ring annuloplasty and $75 \%$ of those with posterior suture bicuspidization remained free of moderate or severe TR $(\mathrm{P}=0.18)(9)$.

\section{Advantages}

The primary anatomic problem underlying functional TR is a dilatation of the posterior tricuspid annulus, which is the least supported area of the tricuspid valve ring (10). As such, the concept of the posterior suture annuloplasty technique is to stabilize this area of the tricuspid valve and annulus. Posterior suture annuloplasty can be performed as a concomitant procedure requiring only $8-10$ additional minutes during the rewarming phase following single or double left sided valve replacement, or even without aortic cross-clamping with procedures such as left ventricular assist device (LVAD) implantation. Primary tricuspid valve pathologies like endocarditis could also be an indication for this surgical technique.

Compared to posterior suture annuloplasty, the earlier techniques have important drawbacks. The DeVega repair has been criticized for its long suture line. This leads to centripetal forces onto both the anterior and posterior annular segments, even though only the posterior segment is primarily responsible for functional TR. Furthermore, its starting point at the anteroseptal commissure may impinge on the atrioventricular node and the bundle of His, risking their injury. The drawbacks of the Kay technique include perpendicular forces on the tricuspid annulus, which leads to excessive deformation of the posterior tricuspid leaflet. This may result in fibrotic transformation of the leaflet. These drawbacks may be avoided by the posterior suture bicuspidization technique.

\section{Caveats}

It is important to note that ring annuloplasty is the procedure of choice for severe functional TR. However, posterior suture annuloplasty is a technically simpler option for patients with moderate functional TR who undergo leftsided valve surgery or LVAD placement. 


\section{Acknowledgements}

None.

\section{Footnote}

Conflicts of Interest: The authors have no conflicts of interest to declare.

\section{References}

1. Dreyfus GD, Martin RP, Chan KM, et al. Functional tricuspid regurgitation: a need to revise our understanding J Am Coll Cardiol 2015;65:2331-6.

2. Kay JH, Maselli-Campagna G, Tsuji HK. Surgical Treatment of Tricuspid Insufficiency Ann Surg 1965;162:53-8.

3. DeVega NF. La anuloplastia selectiva, regulable y permanente Rev Esp Cardiol 1972;25:555-6.

4. Carpentier A, Deloche A, Hanania G, et al. Surgical management of acquired tricuspid valve disease J Thorac Cardiovasc Surg 1974;67:53-5.

5. Filsoufi F, Chikwe J, Carpentier A. Rationale for

Cite this article as: Tchantchaleishvili V, Rajab TK, Cohn LH. Posterior suture annuloplasty for functional tricuspid regurgitation. Ann Cardiothorac Surg 2017;6(3):262-265. doi: 10.21037/acs.2017.05.04 remodelling annuloplasty to address functional tricuspid regurgitation during left-sided valve surgery. Eur J Cardiothorac Surg 2015;47:1-3.

6. Khorsandi M, Banerjee A, Singh H, et al. Is a tricuspid annuloplasty ring significantly better than a DeVega's annuloplasty stitch when repairing severe tricuspid regurgitation? Interact Cardiovasc Thorac Surg 2012;15:129-35.

7. Cohn LH. Tricuspid regurgitation secondary to mitral valve disease: when and how to repair J Card Surg 1994;9:237-41.

8. Navia JL, Nowicki ER, Blackstone EH, et al. Surgical management of secondary tricuspid valve regurgitation: annulus, commissure, or leaflet procedure? J Thorac Cardiovasc Surg 2010;139:1473-1482.e5.

9. Ghanta RK, Chen R, Narayanasamy N, et al. Suture bicuspidization of the tricuspid valve versus ring annuloplasty for repair of functional tricuspid regurgitation: midterm results of 237 consecutive patients J Thorac Cardiovasc Surg 2007;133:117-26.

10. DeLoche A, Guerinon J, Fabiani JN, et al. Étude anatomique des valvulopathies rheumatismales tricuspidiennes Ann Chir Thorac Cardiovasc 1973;4:32-7. 